\title{
How Teachers Solve Students' Conflicts with Emotional Regulation Strategies
}

\author{
Yi Huang* \\ School of Psychology, University of British Columbia, Vancouver, BC, V6T 1Z4, Canada \\ *joyhy0913@gmail.com
}

Keywords: emotional conflict, emotional regulation, emotional regulation strategies, teacher support

\begin{abstract}
Children always face conflicts, especially emotional conflicts during their development because of their low self-control ability. Learning appropriate methods to deal with different situations and conflicts, which is referred to as emotion regulation, is essential to improve the academic performances and social competence of children. Therefore, this systematic review is interested in how children get emotional support from their teachers, who seem to always play important roles in each students' life. The review is intended to find some common-used strategies from teachers to help children with emotional regulation, and whether a specific method is more efficient than others. Although there are differences in the age and gender in terms of children's emotional regulation, based on the overall tendency of childhood development, the four categories of emotional regulation strategies are widely used by teachers: distraction, comfort, cognitive reappraisals, and instrumental behaviour. Distraction is to shift the attention from the original stimulus by providing a distracting stimulus; comfort is defined as the physical behavior such as hugging, holding or verbally saying "it's okay"; cognitive reappraisals refer to the reappraisals of the stressors through the change of thinking perspective; instrumental behavior is the attempt to change the situation or even exclude the possible source of frustration. Moreover, current research has indicated that the socialization of emotional strategies, such as cognitive reframing, is more effective than physical comfort. However, teachers should still pay attention to the cultural difference in emotional regulation and develop a sense of emotional labour to prepare for uncertain situations.
\end{abstract}

\section{Introduction}

Students' conflicts are a universal phenomenon on campus [1]. School is thought to be a simulated society where children can learn, especially learn how to manage their emotions and develop their emotional competence when they are interacting with people. Since children have a relatively lower ability of self-control, their emotions are easily influenced by the environment, they need more neural efforts to deal with emotional conflicts. Emotional conflict here does not refer to emotional diseases but some opposing or extreme feelings relating to a recent situation. Anger, sadness and tension are the most typical expressions that children will show in conflicts [2]. Sometimes it may also accompany some physical discomforts, such as headaches, during the age of childhood and adolescence.

Practitioners and academia stated that emotion regulation, a social-emotional competence to change expression, could help students to deal with those challenging situations [3]. Eisenberg and Morris [4] defined emotion regulation as "the process of initiating, avoiding, inhibiting, maintaining, or modulating the occurrence, form, intensity, or duration of internal feeling states, emotion-related physiological processes, emotion-related goals, and/or behavioral concomitants of emotion, generally in the service of accomplishing one's goals”.

Meanwhile, teachers are responsible for cultivating students' emotional regulation because children are dependent on guidance from adults, especially teachers and children spend almost half of their day in the school. Through warmth, respect, sensitivity, responsiveness and individualization, teachers create and maintain a conducive relationship with students. Thus, it 
increases students' social competence, child autonomy and bulwark. The advice and method of teachers provided to improve children's regulation capacities could have a long influence on their future success. It is also argued that emotional support from teachers could help children to adapt to school life better with appropriate school adjustment, which refers to positive behaviors and attitudes in the classroom context and successful interaction with peers and teachers [5]. Moreover, teachers cultivate children's emotion knowledge, which is the emotional competence influence on children's school-related competence, could help improve children's academic performance and a positive predictor of the teacher-student relationship and school adjustment [6].

Due to the importance of the teacher's role, it is necessary to re-evaluate the quality and competence of teachers and their methods used to modify emotions of children (i.e. emotion-focused teacher-training and reflective practices) [7]. One of the goals of teachers is to help children build up emotional competence, similar to emotional regulation, which is a term about how children apply their knowledge of emotion to manage their emotional expression and negotiate social exchanges.

However, due to inability, and lack of awareness or proper training, teachers may have a negative attitude towards children and their behavior, and these unconscious attributions of teachers may damage children's future development physically and psychologically. As a consequence, there is emergence for teachers to develop their social-emotional competence to regulate behaviors and maintain a supportive teacher-children relationship and effective classroom learning. The strategies could be used by teachers to help children with emotional regulation are similar to the self-reported measure, such as cognitive restructuring, information seeking, problem-solving, emotional ventilation, acceptance, distraction, humor, social withdrawal, denial, and so on.

Therefore, this research is going to_study the teacher's effective strategy to manage children's emotions in conflict situations through discourse analysis. This article aims to facilitate a better understanding on managing children's unstable emotion when some unexpected contradiction happens, and on importance of the role teachers that plays in this interaction so that multiple choices of strategy are provided to manage children's emotion better in conflicts.

\section{Emotional Conflicts}

Emotional conflicts are common among children because they are more impulsive and easily controlled by negative emotions. Conflict control is an essential part of cognitive control and self-regulation during childhood. These conflicts situations contain both emotional conflicts and non-emotional (physical) conflicts. The difference in the change of the volume of gray matter and white matter during the age of adolescence suggests that characteristics of them thinking impulsively and behaving more irrationally could account for the occurrence of conflicts. Therefore, the emphasis on emotional development could not be ignored, Emotional conflict here is some opposing and extreme emotions in stressful situations, and anger and sadness are the most typical emotional expression that children will show in conflicts situation. Children are lack of self-regulation, so sometimes they would express their emotions in some extremely negative ways or have emotion dysregulation. Research indicates that students, who tend to have negative emotional expressiveness, have significant problems getting along with their peers [8]. It is also a negative predictor for an academic barrier, which is the lower academic performance at school.

\section{Emotion Regulation}

Conflict resolution, which refers to the ability to deal with challenging situations, has been found to be associated with adolescent psychosocial adjustment. The research found that when the situations require children to show their emotion regulation strategies, they are more confused and overwhelmed, and it is a strong predictor of the negative relationship with their friends and teacher rating feedback. Emotion regulation is the ability to adjust the feelings according to the situation, and always need to be combined with strategies that could improve efficiency. It is a blend of the internal and external process when children take efforts to initiate, maintain and modulate the 
occurrence, intensity, and expression of emotions. Based on the characteristics of the development of emotion regulation from infancy to late childhood, the most helpful methods for young students are external influence, such as the emotional support from teachers.

\subsection{Characteristics}

At the stage of childhood, there is a change in reliance from the outside world to intra-organismic regulation, and the internal cognitive strategies could be developed through the practice of selecting appropriate strategies for different stressors. The adaptive ("I calm down first and then rethink again") and social support seeking ("I need to talk to related people") had a U-shaped change during followed by an increase for emerging adulthood. Moreover, emotional stability is low during adolescence because of the change in cognitive and biological development, and they often face a lot of conflicts with parents and teachers, which leads to an increase of negative emotion in daily life. There are also gender differences among the emotion regulation problems that males used more negative peer conflict resolution strategies than females did [9]. Female participants have more social support seeking ("I need advice and talk")and dysfunctional rumination ("I think about it all the time") to manage their emotion, while male reported more passivity ("I just wait”), avoidance ("I just go away"), and expressive suppression ("I do not show how I feel”). It is not a specific problem for a certain age but a commonplace in all school experiences, and significant differences were found between students in different grade levels.

However, commons still could be found. There are no changes in the expressive suppression ("I hide my feeling") from the early adolescent to late adolescence. Specific teaching strategies of emotion regulation likely have a strong impact on overall emotional competence because such practice could teach children more regulatory skills and strategies. Emotion regulation has already begun in childhood. In early adulthood, expressed anger may decrease while the depression might increase, so there is more use of passive emotion strategies, such as suppression, avoidance-denial pattern, but less use of active regulation strategies, like emotional copying or seek social support. The reason for emotion dysregulation surprisingly is not the absence of emotion regulation, but due to the deviation of successful regulation techniques.

\subsection{Emotional Support from Teacher}

School is a place to learn, especially emotion competence (i.e. self-confidence, self-esteem, self-regulation, social interaction and empathy). Children could apply the abilities of their knowledge to manage their emotions and deal with negative conflicts with others, which could be related to students' behaviors and attitudes, skills and knowledge, academic success, etc. For most children, preschool represents a period of learning how to navigate the challenges of the classroom for the first time, often creating a trajectory for success or failure in later academic contexts.

When children cannot manage their emotions by themselves, it is important for them to get help from teachers to deal with conflict situations. Classrooms act as a social place where interaction with the children and the teacher should take responsibility to help children with emotion regulation since emotion needs to be practiced in a social context. The research found that teacher help and support had a positive impact on student conflict resolution behaviors and feelings because it could provide a sense of safety and warmth to students.

Two broad theories lay a foundation for emotional support in educational contexts: attachment theory and self-determination theory. For attachment theory, children have their freedom to explore and be influenced by the warm, safe, and consistent environment that their teachers created. For self-determination theory, children are internally motived to learn when their autonomy is supported and their competencies are targeted and reinforced. A model is suggested by Bergin and Bergin that children's attachment to teachers is associated with their school success because the improved emotion regulation abilities, in turn, support their academic achievement through social-emotional competence.

The conflict resolution is related to the extent of how teachers understand the student conflicts, and the well-being of students. Moreover, the consistency and speed that take teacher response could enhance a safe inner world built by teachers. As for the aspects of the emotional support of 
teachers, such as warmth, sensitivity, responsiveness and child-centeredness among the teacher-children relationship, these characters are crucial to the school adjustment of children in early childhood since the impression of school could have a long-term developing effect. However, the unsupportive or lacks of supports will damage children's development. Students presented less self-regulation under the classroom with indifference from teachers or teacher-centered learning model.

\section{Strategies of Emotion Regulation}

It is possible that some emotion regulations are better than others because they could improve the regulation skills of children further. However, the cultural difference in terms of emotion regulation sometimes makes it hard to decide. Since the cultural norms of Western encourage individualism, the children are more likely to express their emotions, while the Eastern countries emphasize interdependence so children always suppress their feelings. Chinese teachers also take a lot of effort to achieve the Confucian philosophy and the ethical norms, which could influence the paradox of the relationship between teacher and students with mixed identities of close and distant and contradictory expression of love and strictness [10].

The scientific mechanism behind the emotional regulation is antecedent person response, referring to the interaction between input (antecedent)- the person- output (response) [11]. Emotion coaching practices contain several behaviors, such as labeling emotions, comfort, and problem-solving that teachers tend to show in response to emotional conflicts of children, which could result in better emotion regulation and understanding of emotions. An important component of emotion coaching is to apply intentional and appropriate strategies for different situations, aiming at helping children regulate their emotions. Some useful strategies of coping with negative such as "taking a deep breath" or "thinking about something else" are commonly suggested. There are mainly four categories of behavior in response to stress and frustration: distraction, comfort, cognitive reappraisals, and instrumental behavior. The attention refocusing, which refers to shift the attention from the original stimulus by providing a distracting stimulus, is effective in reducing anger but not fear. Moreover, the active problem-solving types of coping works are better than passive coping and rumination. Comforting is widely used as a physical behavior to regulate the children's emotions, such as hugging, holding or verbally saying "it's okay" by not changing the situations but tend to be less effective with the increase of age. By contrast, cognitive reframing is the reappraisals of the stressors through the change of perspective, and it happens when the situations are interpreted differently through reframing so the negative consequences are not existing. Instead of thinking "half empty" with negative emotions, it is better to believe it is "half full" with positive feeling and optimism, though the meaning is exactly the same. Instrumental strategies are the attempt to change the situation or even exclude the possible source of frustration, which is harder to achieve. It is believed that attention refocusing and cognitive reframing are more effective strategies to deal with the negative emotion of children because they are socialization efforts that aimed at more cognitive coping strategies rather than_physical strategies, and these methods could also be applied to teacher-children relationship.

As a result, sometimes it could be difficult for teachers to select the most suitable method on emotion regulation; they tend to be depressed and frustrated when they face the non-functional response of children. Therefore, it is important for them to have emotional labor to adjust their own emotion. Emotional labor strategies are to express appropriate emotions, although that would be inconsistent with their inner one. Emotional labor requires teachers to improve their ability to regulate their emotions (burden out, well-being, job satisfaction, etc.), otherwise emotional exhaustion, and the process by which teachers inhibit, generate, and manage their feelings and expression of emotions, according to normative beliefs and expectations.

\section{Conclusion}

Children always face conflicts, especially emotional conflicts during their development. It is 
found that conflicts can be solved by adolescent psychosocial adjustment, which refers to emotion regulation. Because children's brains are not fully developed, they are more vulnerable to the influence from their environment. Therefore, they are more dependent on the help of adults, especially the emotional support from their teachers at school due to the possible long effect on the future and success of school adjustment. Although there are differences in the age, gender and culture of the features of emotional regulation, based on the overall tendency of childhood development, the four emotional regulation strategies of distraction, comfort, cognitive reappraisals, and instrumental behavior are typically used. The socialization of emotional strategies, such as cognitive reframing, is more effective than physical comfort. However, teachers should still pay attention to the cultural difference on emotional regulation and develop emotional labor to prepare for uncertain situations.

\section{References}

[1] Liu, T., Liu, X., Li, D., Shangguan, F., Lu, L., \& Shi, J. "Conflict control of emotional and non-emotional conflicts in preadolescent children”. Biological Psychology, 146, pp.107708, 2019;

[2] Morris, A. S., Silk, J. S., Morris, M. D. S., Steinberg, L., Aucoin, K. J., \& Keyes, A. W. “The influence of mother-child emotion regulation strategies on children's expression of anger and sadness”. Developmental Psychology, 47(1), pp.213-225, 2011.

[3] Bailey, C. S., Denham, S. A., Curby, T. W., \& Bassett, H. H. "Emotional and organizational supports for preschoolers' emotion regulation: Relations with school adjustment". Emotion (Washington, D.C.), 16(2), pp.263-279, 2016;

[4] Eisenberg, N., \& Morris, A. S. "Children's emotion-related regulation. Advances in Child Development and Behavior”. 30, pp.189, 2002.

[5] Herndon, K. J., Bailey, C. S., Shewark, E. A., Denham, S. A., \& Bassett, H. H. "Preschoolers' emotion expression and regulation: Relations with school adjustment”. The Journal of Genetic Psychology, 174(6), pp.642-663, 2013.

[6] Garner, P. W., \& Waajid, B. "The associations of emotion knowledge and teacher-child relationships to preschool children's school-related developmental competence”. Journal of Applied Developmental Psychology, 29(2), pp.89-100, 2008.

[7] Ulloa, M., Evans, I., \& Jones, L. "The effects of emotional awareness training on teachers' ability to manage the emotions of preschool children: An experimental study". Escritos De Psicología (Internet), 9(1), pp.1-14, 2016.

[8] Rakap, S., Balikci, S., Kalkan, S., \& Aydin, B. "Preschool teachers' use of strategies to support social-emotional competence in young children”. International Journal of Early Childhood Special Education, 10(1), pp.11-25, 2018.

[9] Zimmermann, P., \& Iwanski, A. "Emotion regulation from early adolescence to emerging adulthood and middle adulthood: Age differences, gender differences, and emotion-specific developmental variations”. International Journal of Behavioral Development, 38(2), pp.182-194, 2014.

[10] Yin, H. "Knife-like mouth and tofu-like heart: Emotion regulation by chinese teachers in classroom teaching”. Social Psychology of Education, 19(1), pp.1-22, 2016

[11] Hu, T., Zhang, D., Wang, J., Mistry, R., Ran, G., \& Wang, X. "Relation between emotion regulation and mental health: A meta-analysis review”. Psychological Reports, 114(2), pp.341-362, 2014. 